Pacific Journal of Mathematic 


\title{
ON ABSOLUTELY TORSION-FREE RINGS
}

\author{
JoRge Viola-Prioli
}

Let $K(R)$ denote the set of the kernel functors of the ring $R$ and let $\infty$ be the trivial kernel functor defined by setting $\infty(M)=M$ for every right module $M$. The absolutely torsion-free rings, that is, the rings $R$ for which $\sigma(R)=0$ for all $\sigma \in K(R), \sigma \neq \infty$, have been introduced by Rubin as a non commutative analogue of the integral domains.

In this paper a categorical characterization (in terms of finitely generated projective modules) of absolutely torsion-free rings is obtained. As a consequence, all of Rubin's results are proved in a different fashion and generalization of most of them are provided. Additional properties of this class of rings are also exhibited.

Finally, absolutely torsion-free rings with torsionless injective hull are also considered.

Preliminaries. Throughout all rings are understood associative and with unity, which is preserved under taking subrings and ring homomorphisms. All modules are unitary right modules. We let $\mathcal{M}_{R}$ denote the category of right $\boldsymbol{R}$-modules.

For any module $M_{R}$ we let $E(M)$ denote an injective hull of $M$. Hence $M$ is large in $E(M)$ and $E(M)$ is injective. If $M$ is a module, $N$ a submodule of $M$ and $S$ a nonempty subset of $M$ we let $\left(N_{R}: S\right)$ denote the right ideal $\{r \in R ; S . r \subset N\}$. When no danger of confusion arises we will simply write $(N: S)$.

The term ideal is reserved to be used for two-sided ideals only. Consequently, a ring is simple if it is has exactly two ideals.

Any unexplained terminology can be found for instance in [10] or [5]. As to the notation and terminology relative to kernel functors, topologizing filters of right ideals, etc, we follow Goldman [6] and Rubin [12] with which familiarity is assumed.

We let $\mathscr{L}$ denote the filter of large right ideals of $R$ and $Z$ its associated kernel functor; consequently $Z(M)$ is the singular submodule of $M$. (See [10].)

The idempotent topologizing filter of dense (or rational) right ideals of $R$ is indicated by $\mathscr{D}$; thus $\mathscr{D} \subset \mathscr{L}$ and they coincide precisely when $Z(R)=0$. (See [8] or [4]). We will write $Q_{\max }(R)$ or simply $Q(R)$ to indicate the maximal ring of right quotients of $R$, that is, the ring of quotients of $R$ with respect to $\mathscr{D}$.

The direct sum (respectively, direct product) of copies of $M_{R}$ taken over a set $\mathscr{I}$ will be denoted by $M^{(\mathfrak{s})}$ (respectively, $M^{\mathcal{s}}$ ); however, if $\mathscr{I}$ has $n$ elements we simply write $M^{(n)}$. 
The absolutely torsion-free rings. As in [12] we set the following.

Definition. $R_{R}$ is called an ATF-ring if and only if $\sigma \in K(R)$, $\sigma \neq \infty$, implies $\sigma(R)=0$.

We start with our basic tool throughout.

Theorem 2.1. For any ring $R$ the following conditions are equivalent :

(1) $R_{R}$ is $A T F$.

(2) The filter $\mathscr{D}$ contains every nontrivial topologizing filter of $R$.

(3) For every finitely generated projective module $P$ and $0 \neq N \subset P$ there exists $f$ and a natural number $n$ (depending on $N$ ) such that

$$
0 \rightarrow P \stackrel{f}{\rightarrow} N^{(n)}
$$

is exact.

(4) For every non zero right ideal I there exist $g$ and a natural number $n$ (depending on I) such that

$$
0 \rightarrow R \stackrel{g}{\rightarrow} I^{(n)}
$$

is exact.

(5) For every nonzero right ideal I there exist $h$ and an indexing set $\Phi$ such that the sequence

$$
I^{(9)} \stackrel{h}{\rightarrow} E(R) \rightarrow 0
$$

is exact.

Proof. (1) implies (2). Set $\tau=\tau_{E(R)}$ (See [6, page 33]). Given $\sigma \in K(R), \sigma \neq \infty$ we have $\sigma(R)=0$. Thus $\sigma(E(R))=0$; therefore $\sigma \leqq \tau$, i.e., $\mathscr{F}(\sigma) \subset \mathscr{F}(\tau)=\mathscr{D}$.

(2) implies (1). Clear.

(1) implies (3). Define $\mathscr{F}=\left\{I_{R} ; \exists k \in \mathbf{N}\right.$ and $m_{1}, \cdots, m_{k}$ in $N$ such that $\left.I \supseteq\left(0: m_{1}\right) \cap \cdots \cap\left(0: m_{k}\right)\right\}$. Then $\mathscr{F}$ is a topologizing filter, say $\mathscr{F}=\mathscr{F}(\sigma)$. Observe that $\sigma(N)=N$. Since $P$ is finitely generated projective we have $P \oplus A \cong R^{(k)}$, for some natural number $k$ and some $A$. Hence $0 \neq N=\sigma(N) \subset \sigma(P) \subset \sigma\left(R^{(k)}\right)$. If $\sigma<\infty$ then $\sigma\left(R^{(k)}\right)=0$, i.e., $N=0$ which is not the case. Therefore $\sigma=\infty$, that is (0) $\in \mathscr{F}$. Thus there exists $\left\{m_{i}\right\} \subset N$ such that

$$
0=\left(0: m_{1}\right) \cap \cdots \cap\left(0: m_{t}\right) .
$$

Define now $\quad \alpha_{1}: R \rightarrow N^{(t)}, \quad \alpha_{1}(1)=\left(m_{1}, \cdots, m_{t}\right) \quad$ and set $\alpha: R^{(k)} \rightarrow N^{(k t)}, \alpha\left(r_{1}, \cdots, r_{k}\right)=\left(\alpha_{1}\left(r_{1}\right), \cdots, \alpha_{1}\left(r_{k}\right)\right)$. Therefore $n=k t$ and 
$f=\left.\alpha\right|_{P}$ will do.

(3) implies (4). Clear.

(4) implies (5). Given such an $I$ we have by assumption the embedding $g: R \rightarrow I^{(n)}$.

For $x \in E(R)$ define $\theta_{x}: R \rightarrow E(R)$ by $\theta_{x}(r)=x \cdot r$, and call $f_{x}$ an extension to $I^{(n)}$. The following sequence

$$
\bigoplus_{x \in E(R)} I^{(n)} \underset{n}{\rightarrow} E(R) \rightarrow 0
$$

where $h=\bigoplus f_{x}$, for all $x \in E(R)$, is exact.

(5) implies (1). Given $\sigma \in K(R), \sigma \neq \infty$ set $I=\sigma(R) . \quad$ If $I=(0)$ we are done. Otherwise, by (5), we find an exact sequence $I^{(s)} \rightarrow E(R) \rightarrow 0$. Clearly $I^{(s)}$ is $\sigma$-torsion and hence $E(R)$ will be so. It follows that $R$ is $\sigma$-torsion, that is, $\sigma=\infty$, a contradiction.

Several observations flow naturally from our characterization of ATF-rings.

COROllary 2.2. Every ATF-ring is prime and right nonsingular. In particular, if $R$ is commutative then $R$ is $A T F$ if and only if is a domain. (See [12] for an alternate proof).

Proof. By (4) above nonzero right ideals of $R$ are faithful and hence $R$ is prime. Also since $(0) \notin \mathscr{L}, \mathscr{L} \subset \mathscr{D}$ and so $Z\left(R_{R}\right)=0$ as asserted. The rest of the corollary is immediate since being a torsion element can be given in terms of annihilating conditions.

Corollary 2.3. Let $R$ be an ATF-ring. Then $R$ is simple artinian if and only if $R$ contains a (nonzero) minimal one sided ideal. Also, $R_{R}$ is noetherian if and only if $R$ contains a nonzero noetherian right ideal.

Proof. If $R$ contains a nonzero minimal left ideal, $\operatorname{soc}\left({ }_{R} R\right) \neq 0$. Since $R$ is a prime ring it follows that $\operatorname{soc}\left(R_{R}\right) \neq 0$. Hence there exists $0 \neq I_{R}$, a simple right ideal. By (4) above we infer that $R$ is semisimple artinian and being prime it is a simple artinian ring. Similarly with noetherianess.

According to this result the converse of (2.2) is false. For $R=$ $\operatorname{End}_{K}(V), V$ a countably infinite dimensional vector space over a field $K$, is primitive (hence prime) and right nonsingular; However it is very well known [7] that $0 \neq \operatorname{soc}\left(R_{R}\right) \neq R$ and so $R$ is not ATF.

Theorem (2.1-3) provides a categorical characterization of ATFrings. Based on that, we obtain in a different way another of Rubin's results. 
THEOREM 2.4. Being an ATF-ring is a Morita invariant.

COROLlaRY 2.5. If $R$ is $A T F$ and $n \geqq 1$, the ring of $n$ by $n$ matrices with coefficients in $R$ is an ATF-ring.

Proof. The rings in question are Morita equivalent rings via the finitely generated projective generator $R^{(n)}$. (See [2]).

If $R$ is ATF it is necessarily right non-singular and consequently $Q(R) \cong E(R)$. (See [8], [10]).

Rubin showed that $Q(R)$ is a simple ring whenever $R$ is ATF. Based on the following three results we can say more.

We first observe that if $R$ is a simple ring and $I_{R} \subset R$ then $I^{2}=I$. Our next result tells us that the reverse implication is true when $R$ is ATF.

Proposition 2.6. Assume that $R$ is ATF. Then, every right ideal equals its square if and only if $R$ is a simple ring.

Proof. Assume $I$ is a nonzero ideal of $R$. Set $E=E(R / I)$ and call $\tau=\tau_{E}$. (See [6, page 33]). We claim that $\tau(I)=I$. In fact, suppose there exists a nonzero homomorphism $f: I \rightarrow E$. Since $R / I \subset^{\prime}$ $E$ we find $x \in I$ such that $f(x) \in R / I$ and $f(x) \neq 0$. Since $x R=(x R)^{2}$ we express $x=x y$ where $y \in I$. It follows that $0 \neq f(x)=f(x y)=$ $f(x) y=0$ since $I$ is an ideal, a contradiction. Thus

$$
I=\bigcap_{g: I \rightarrow E}(\text { kernel } g)
$$

that is, $\tau(I)=I$. Since $I \neq(0), \tau=\infty$ by hypothesis. Hence $E=0$, which forces $I$ to equal $R$. Therefore $R$ is simple, as asserted.

As in [4] $R$ is called a (right) $V$-ring if every simple right module is injective.

COROLlary 2.7. Let $R$ be ATF. If $R$ is regular or a $V$-ring then it is simple.

Proof. It is easy to see that in a $V$-ring every right ideal equals its square. The same is true if $R$ is regular. Apply previous proposition.

Every ring between a commutative domain and its field of quotients is obviously a domain. The analogue for ATF-rings is the following.

PROPOSITION 2.8. Let $R$ be $A T F$ and $S$ a ring such that $R \subset S \subset$ $Q(R)$. Then $S$ is $A T F$. (See [12] for an alternate proof). 
Proof. Given $s \in S, s \neq 0$, pick $r \in R$ such that $s r \in R$ and $s r \neq 0$. Set $r^{*}=s r$. By (2.1) we can find $x_{1}, \cdots, x_{k}$ in $R$ such that

$$
0=\bigcap_{j=1}^{k}\left(0 ; r_{i}^{*} x_{j}\right)
$$

We claim that for every $a \in S, s\left(r x_{j}\right) a=0$ for all $1 \leqq j \leqq k$, implies $a=0$. In fact, $s\left(r x_{j}\right) a R=0$ for all $j$ implies $s\left(r x_{j}\right) \cdot(a R \cap R)=0$ and so

$$
a R \cap R \subset \bigcap_{j=1}^{k}\left(0: r_{R}^{*} x_{\jmath}\right)=0 .
$$

Therefore $a R \cap R=0$, i.e., $a=0$ as $R_{R} C^{\prime} S_{R}$.

We have shown that $0=\cap_{j=1}^{k}\left(0: s\left(r x_{j}\right)\right)$, which implies that $S$ is ATF according to $(2.1-4)$.

Corollary 2.9. Let $R$ be ATF, $S$ a ring such that $R \subset S \subset$ $Q(R)$. If $S$ is regular or a right $V$-ring then $S$ is simple. In particular, $Q(R)$ will be a simple ring.

The usual localization (at a multiplicatively closed subset) of a commutative domain is a domain. The analogue for ATF-rings is next.

A right localization of a ring $R$ is a pair $(\phi, S)$ where $S$ is a ring, $\phi: R \rightarrow S$ is an epimorphism in the category of rings with unity and $S$ is flat as a left $R$-module in a natural way, via $\phi$. (See [9], [13].)

Corollary 2.10. Let $R$ be an ATF-ring and $\phi: R \rightarrow S$ a right localization. Then $S$ is an ATF-ring.

Proof. It follows from [11] that $S \cong Q_{\nu}(R)$ as rings, where $\nu \in I(R)$ and $\mathscr{F}(\nu)=\left\{I_{R} ; \phi(I) \cdot S=S\right\}$. Since $\nu<\infty, \nu(R)=0$ by hypothesis. By definition of $Q(R)$ we have a ring embedding

$$
R \rightarrow Q_{\nu}(R) \rightarrow Q(R)
$$

and therefore $S$ is ATF in virtue of (2.8).

The converse of this result is false, i.e., there exists a ring such that every nontrivial right localization is ATF but the ring itself is not so. As a simple example consider a field $F$ and set

$$
R=\left\{\left[\begin{array}{cc}
F & F \\
0 & F
\end{array}\right]\right\}
$$

$R$ is a nonsingular ring and

$$
I(R)=\{0, Z, \nu, \infty\}
$$


where $\nu\left(M_{R}\right)=\{m \in M ; m \cdot I=0\}$ and $I=\left\{x \in R ; x_{22}=0\right\}$.

We have that $Q_{z}(R)=Q(R)=M_{2 \times 2}(F)$ and $Q_{\nu}(R) \cong F$ since $R / \nu(R)=R / L$, where $L=\left\{x \in R ; x_{11}=0\right\}$ and so $R / L$ is in fact an injective $R$-module. It follows that all nontrivial localizations of $R$ are ATF; however $R$ is not even prime.

As Rubin observed, subrings of ATF need not be ATF. (For instance, consider $R$ as defined in our preceding remark). There is, however, a particular instance in which ATF-ness is recovered.

Lemma 2.11. If $R$ is a right order in an ATF-ring $A, R$ is $A T F$.

Proof. For any $r \in R, r \neq 0$, there exist $a_{1}, \cdots, a_{n}$ in $A$ such that

$$
0=\bigcap_{i=1}^{n}\left(0: r a_{i}\right) \text {. }
$$

It is well known that we can find a common denominator for the $a_{i}$ 's, i.e., there exists a regular element $d \in R$ such that for some $r_{i} \in R$, $1 \leqq i \leqq n, a_{i} d=r_{i}$. If $r^{*} \in R$ is such that $r r_{i} r^{*}=0$ for $1 \leqq i \leqq n$ then $0=r a_{i} d r^{*}$ for all $i$. Accordingly, $d r^{*}=0$ and hence $r^{*}=0$. Therefore $0=\cap_{i=1}^{n}\left(0: r r_{i}\right)$, which shows that $R$ is ATF, as claimed.

A theorem of Faith [4, page 130] states that any prime right Goldie $V$-ring is simple. This is a consequence of our next

PROPOSITION 2.12. A right $V$-ring $R$ which is a right order in an ATF-ring, is necessarily simple.

Proof. According to (2.11) $R$ is ATF. That $R$ is simple follows therefore from (2.7).

Our next goal is to produce more ATF-rings. We need to recall the following

DEFINITION. A semigroup $\left(S,{ }^{\circ}\right)$ is said to be simply linearly ordered if it admits a linear ordering " $\leqq$ " such that $s \leqq t, u \leqq v$ implies $s \circ u \leqq t \circ v$.

The following result is very well known. Seemingly, our proof is not in the literature.

LemMa 2.13. Any torsion-free abelian group can be simply linearly ordered.

Proof. If $G$ is a torsion-free abelian group, necessarily is flat. Therefore we obtain the following exact sequence of abelian groups:

$$
0 \rightarrow G \cong G \otimes Z \stackrel{f}{\rightarrow} G \otimes Q
$$


Since $G \otimes Q$ is a $Q$-vector space there exists a set $\mathscr{I}$ such that $\boldsymbol{G} \otimes \boldsymbol{Q} \cong \boldsymbol{Q}^{(\boldsymbol{G})}$. After well ordering $\mathscr{I}$ we define in $\boldsymbol{G} \otimes \boldsymbol{Q}$ the lexicographic order, which clearly makes it into a simply linearly ordered abelian group. Therefore, by mean of $f, G$ becomes a simply linearly ordered abelian group.

Proposition 2.14. Let $R$ be an ATF-ring and $(S, \cdot)$ a simply linearly ordered cancellative semigroup. Then, the semigroup ring $R[S]$ is $A T F$.

Proof. Let $a \neq 0$ be an element in $A=R[S]$, say

$$
a=r_{1} s_{1}+\cdots+r_{n} s_{n}
$$

where $s_{1} \neq s_{2} \neq \cdots \neq s_{n}$ and $0 \neq r_{i}$ for $i=1, \cdots, n$.

Set next $r_{n}=r$. We know that for some $x_{1}, \cdots, x_{k}$ in $R, 0=\cap_{i=1}^{k}$ $\left(0: r x_{i}\right)$. We now claim that

$$
0=\bigcap_{j=1}^{k}\left(0: r x_{i}\right)
$$

In fact, assume there exists $a^{*} \in A$, such that $a x_{i} a^{*}=0$ for all $1 \leqq i \leqq k$. Write $a^{*}=d_{1} t_{1}+\cdots+d_{p} t_{p}$ with $d_{i} \in R, t_{i} \in S, t_{1}<\cdots<t_{p}$ and all the $d_{i}$ 's different from zero. Since $s_{m} t_{q}<s_{n} t_{p}$ unless $m=n$ and $q=p$ we obtain $0=r x_{i} d_{p}$ for $i=1, \cdots, k$. Hence, $d_{p}=0$. Conclude that $a^{*}=0$ as asserted and accordingly $R[S]$ is ATF.

\section{Corollary 2.15. Assume $R$ is ATF. Then}

(a) $R[G]$ is $A T F$ if $G$ is a torsion-free abelian group.

(b) $R\left[x_{i}\right]_{i \in I}$, the polynomial ring on any number of indeterminates, is $A T F$.

REMARKS. (1) In (2.14) the cancellativity of $S$ is needed as can be seen by considering a field $F, S$ a semigroup formed by two different elements $a$ and $b$ with the multiplication law given by: $a b=b a=b^{2}=$ $a^{2}=a$ and by declaring $a \leqq b$. Hence, $S$ is simply linearly ordered but is not cancellative. The ring $R=F[S]$ is not ATF since (2.1-4) fails when $I=a \cdot R$.

(2) We do not know what conditions on a group $G$ assures the ATF-ness of the group ring $R[G]$. A theorem of Connell [10, page 165] states that if $G$ is a group then $R[G]$ is prime if and only if $R$ is prime and $G$ has no nonzero finite normal subgroups. We therefore have that if $R[G]$ is ATF then $R$ is ATF (a simple computation) and since Connell's theorem takes over, $G$ has no nonzero finite normal subgroups. To see that the reverse implication is false take a suitable infinite permutation group and quote [10, Proposition 2, page 155]. 
ATF-rings with torsionless injective hull. As in [3] $M_{R}$ is said to be torsionless whenever there exists an indexing set $\mathscr{I}$ and an embedding $f: M_{R} \rightarrow R^{s}$.

It is known that if $R$ is a domain or a simple ring or a prime Goldie ring and if $E\left(R_{R}\right)$ is a torsionless $R$-module then $R$ and $E(R)$ coincide. Armendariz [1] investigated prime right nonsingular rings with torsionless injective hull. Our aim in this section is to study under what conditions an ATF-ring with torsionless injective hull is necessarily right selfinjective. Our result must be compared to that one of Armendariz.

We will make use of the following very well known result due to Zelmanowitz [14].

Lemma 3.1. Let $E_{R}$ be an injective module, $M_{R}$ a nonsingular module and $f: E \rightarrow M$ an R-homomorphism. Then $f$ splits. In particular, if $f$ is onto, $M$ is injective.

The main result of this section is the following

THEOREM 3.2. Let $R_{R}$ be $A T F$ and $Q(R)$ torsionless. Then every ideal of $R$ which is left finitely generated is also right finitely generated if and only if $R=Q(R)$.

Proof. Set $Q=Q(R)$. Since $Q$ embeds into $R^{\mathscr{S}}$ for some indexing set $\mathscr{I}$, we obtain a nonzero projection $p: Q \rightarrow R$. According to (3.1) $p(Q)=I_{R}$ is injective. Since $R$ is ATF there exists $n \in N$ and an embedding $f: R \rightarrow I^{(n)}$; the injectivity of $I^{(n)}$ gives therefore a monomorphism from $Q$ into $I^{(n)}$. Since $Q=E(R)$ (because $Z(R)=0$ ) conclude that $Q_{R}$ is finitely generated projective. Write next $T=$ Trace ideal of $Q_{R}=\Sigma_{g \in Q^{*}} g(Q)$, where $Q^{*}=\operatorname{Hom}_{R}(Q, R)$. Observe that if $g \in Q^{*}$ then $g$ is a $Q$-homomorphism. Therefore $T=$ $\{r \in R ; r Q \subset R\}$.

Set $V=\Sigma I_{\gamma}$, for all injective right ideals $I_{\gamma} \subset R$. According to (3.1) $T \subset V$. On the other hand, if $I_{\gamma}$ is injective, the projection $R \rightarrow I_{\gamma}$ extends to an onto map $Q \rightarrow I_{\gamma}$ and so $V \subset T$. Hence $T=V$.

Since $Q_{R}$ is finitely generated projective, say $Q=q_{1} R+\cdots+q_{n} R$, by the dual basis lemma we can find $f_{i} \in Q^{*}, 1 \leqq i \leqq n$ such that $1=q_{1} f_{1}(1)+\cdots+q_{n} f_{n}(1)$. Clearly, for $i=1, \cdots, n$, we have $f_{i}(1) \in T$ and if $t \in T$ then $t=\sum_{i=1}^{n}\left(t q_{i}\right) \cdot f_{i}(1)$ with $t q_{i} \in R$ for $i=1, \cdots, n$. It follows that $T$-which is an ideal-is left finitely generated and hence, by hypothesis, is right finitely generated, which allows us to write it as $I_{1}+\cdots+I_{m}$, where the $I_{j}$ 's are injective right ideals of $R$. It follows from (3.1) that $T$ is injective, being image of the injective module $I_{1} \oplus \cdots \oplus I_{m}$. There exists an idempotent $e$ in $R$ such that $T=e R$ and so $1-e=0$ since $R$ is prime. Conclude that $T=R$ and hence $R_{R}$ is injective, as claimed. 
The other implication is clear since $R$ turns out to be regular and hence simple according to (2.7).

Remark. After this work was completed D. Handelman constructed a non-self injective ATF-ring with torsionless (in fact, projective) maximal ring of right quotients.

\section{REFERENCES}

1. E. Armendariz, Torsion free rings with torsionless injective hull, Preprint.

2. H. Bass, The Morita theorems, Lecture notes published by the Department of Mathematics, Univ. of Oregon (1962).

3. - Finitistic dimension and a homological generalization of semiprimary rings, Trans. Amer. Math. Soc., 95 (1960), 466-488.

4. C. Faith, Lectures on injective modules and quotient rings, Lecture Notes in Mathematics No. 49, Springer-Verlag, Berlin and New York, 1967.

5. — Algebra: Rings, Modules and Categories, Springer-Verlag, New York and Berlin, 1973.

6. O. Goldman, Rings and modules of quotients, J. Algebra, 13 (1969), 10-47.

7. N. Jacobson, Structure of rings, Amer. Math. Soc., Providence, R. I., 1964.

8. R. Johnson, Quotient rings of rings with zero singular ideal, Pacific J. Math., 11 (1961), $1385-1392$.

9. J. Knight, On epimorphisms of non commutative rings, Proc. Cambridge Phil. Soc., 68 (1970), $589-600$.

10. J. Lambek, Lectures on rings and modules, Blaisdell Publishing Co., Waltham, Mass., 1966.

11. N. Popescu and T. Spircu, Sur les épimorphismes plats d'anneaux, C. R. Acad. Sci. Paris, 268 (1969), 376-379.

12. R. Rubin, Absolutely torsion-free rings, Pacific J. Math., 2 (1973), 503-514.

13. L. Silver Non commutative localization and applications, J. Algebra, 7 (1967), 44-76.

14. J. Zelmanowitz, Endomorphism rings of torsionless modules, J. Algebra, 5 (1967), 325-341.

Received October 25, 1973 and in revised form September 3, 1974. This paper is based on a portion of the author's doctoral dissertation at Rutgers, The State University. The author is deeply indebted to his thesis advisor, Professor Barbara L. Osofsky, for the constant encouragement provided during his studies and for her generous help during the organization of this material. 




\section{Pacific Journal of Mathematics}

\section{Vol. 56, No. $1 \quad$ November, 1975}

Shimshon A. Amitsur, Central embeddings in semi-simple rings .......... 1

David Marion Arnold and Charles Estep Murley, Abelian groups, A, such

that $\operatorname{Hom}(A,---)$ preserves direct sums of copies of $A \ldots \ldots \ldots .$.

Martin Bartelt, An integral representation for strictly continuous linear

operators ................................... 21

Richard G. Burton, Fractional elements in multiplicative lattices......... 35

James Alan Cochran, Growth estimates for the singular values of

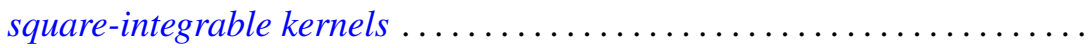

C. Martin Edwards and Peter John Stacey, On group algebras of central

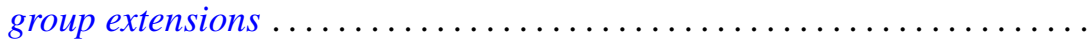

Peter Fletcher and Pei Liu, Topologies compatible with homeomorphism

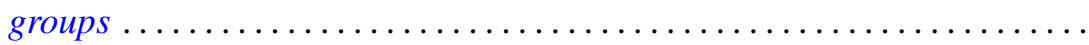

George Gasper, Jr., Products of terminating ${ }_{3} F_{2}(1)$ series ............ 87

Leon Gerber, The orthocentric simplex as an extreme simplex ............

Burrell Washington Helton, A product integral solution of a Riccati

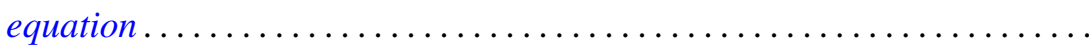

Melvyn W. Jeter, On the extremal elements of the convex cone of

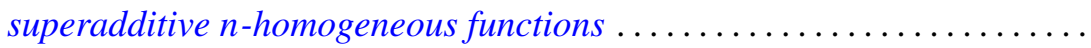

R. H. Johnson, Simple separable graphs .

Margaret Humm Kleinfeld, More on a generalization of commutative and

alternative rings. . .

A. Y. W. Lau, The boundary of a semilattice on an $n$-cell.

Robert F. Lax, The local rigidity of the moduli scheme for curves ...

Glenn Richard Luecke, A note on quasidiagonal and quasitriangular

operators .

Paul Milnes, On the extension of continuous and almost periodic functions

Hidegoro Nakano and Kazumi Nakano, Connector theory.

James Michael Osterburg, Completely outer Galois theory of perfect rings ..................................

Lavon Barry Page, Compact Hankel operators and the F. and M. Riesz

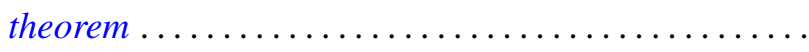

Joseph E. Quinn, Intermediate Riesz spaces..................... 225

Shlomo Vinner, Model-completeness in a first order language with a generalized quantifier.

Jorge Viola-Prioli, On absolutely torsion-free rings ..........

Philip William Walker, A note on differential equations with all solutions of integrable-square............................

Stephen Jeffrey Willson, Equivariant maps between representation 